\title{
Experimental implementation of the optimal linear-optical controlled phase gate
}

\author{
K. Lemr, ${ }^{1}$ A. Černoch, ${ }^{1}$ J. Soubusta, ${ }^{1}$ K. Kieling, ${ }^{2}$ J. Eisert,,${ }^{2,3}$ and M. Dušek ${ }^{4}$ \\ ${ }^{1}$ Joint Laboratory of Optics of Palacký University and Institute of Physics of \\ Academy of Sciences of the Czech Republic, 77907 Olomouc, Czech Republic \\ ${ }^{2}$ Institute of Physics and Astronomy, University of Potsdam, 14476 Potsdam, Germany \\ ${ }^{3}$ Institute for Advanced Study Berlin, 14193 Berlin, Germany \\ ${ }^{4}$ Department of Optics, Faculty of Science, Palacký University, 77146 Olomouc, Czech Republic
}

(Dated: November 10, 2018)

\begin{abstract}
We report on the first experimental realization of optimal linear-optical controlled phase gates for arbitrary phases. The realized scheme is entirely flexible in that the phase shift can be tuned to any given value. All such controlled phase gates are optimal in the sense that they operate at the maximum possible success probabilities that are achievable within the framework of any postselected linear-optical implementation. The quantum gate is implemented using bulk optical elements and polarization encoding of qubit states. We have experimentally explored the remarkable observation that the optimum success probability is not monotone in the phase.
\end{abstract}

PACS numbers: 42.50.Ex, 03.67.Lx

Linear-optical architectures belong to the most prominent platforms for realizing protocols of quantum information processing [1, 2]. In small-scale applications of quantum information, such as in quantum repeaters, they will quite certainly play a key role. Unsurprisingly, a significant research effort has been dedicated in recent years to experimental realization of universal linear-optical quantum gates. Linearoptical quantum gates are probabilistic by their very nature [1]. Therefore, the exact trade-offs between properties of a gate, such as entangling power, and its probability of success are in the focus of attention.

In this work, we explore this trade-off for the first time experimentally. We present data from an experimental realization of a linear-optical, post-selected controlled phase gate implementing the following operation on two qubits:

$$
\begin{aligned}
|0,0\rangle & \mapsto u_{0,0}|0,0\rangle=|0,0\rangle, \\
|0,1\rangle & \mapsto u_{0,1}|0,1\rangle=|0,1\rangle, \\
|1,0\rangle & \mapsto u_{1,0}|1,0\rangle=|1,0\rangle, \\
|1,1\rangle & \mapsto u_{1,1}|1,1\rangle=e^{i \varphi}|1,1\rangle,
\end{aligned}
$$

for an arbitrary given phase $\varphi \in[0, \pi]$. It is key to this experiment that this angle can be chosen in a fully tunable fashion, hence adding a flexible scheme to the linear optical quantuminformation-processing toolbox.

Controlled phase gates are important members of this toolbox. For example, they play a key role in the circuit for quantum Fourier transform [3]. They are entangling quantum gates in general and, together with single-qubit operations, they form a universal set for quantum computing. Notice that the controlled-NOT gate can be obtained by applying a Hadamard transform to the target qubit before and after a controlled phase gate with phase shift $\pi$.

Previous experimental work was devoted to the linearoptical realization of a special case of the controlled phase gate with the fixed phase $\varphi=\pi$ [4]. Ref. [5] presents an experiment with phases different from $\pi$, but with a non-optimal probability of success. The optimal success probability has recently been identified theoretically in Ref. [6]. This opti- mum probability we have indeed reached in the experiment described in this Letter. We observe the quite remarkable trade-off between the entangling power of the gate and its success probability, which is-surprisingly-not monotonous in the phase on $[0, \pi]$. The success probability decreases rapidly for small phases, but remains almost constant for phases between $\pi / 4$ and $\pi$. This experiment is hence expected to be both interesting conceptually as well as technologically, since a fully tunable bulk linear-optical architecture is presented, uplifting tunable schemes for quantum state preparation [7] to the level of quantum information processing.

Theoretical framework: We start by introducing the theoretical framework underlying the experiment. For postselected linear optical gates, the beam splitter matrix $A$ describing a general linear optics network is constrained by the action of the gate (1) as

$$
\operatorname{per} A\left[c_{i}, c_{j} \mid c_{k}, c_{l}\right]=u_{i, j} \delta_{i, k} \delta_{j, l}
$$

where $i, j, k, l=0,1$. The left hand side is the permanent of a matrix filled by matrix elements of $A$ [8]. $c_{0}=(0,1), c_{1}=$ $(1,0)$ are vectors describing the usual dual-rail encoding into Fock states on two modes [9]. Due to post-selection, only outcomes in the computational subspace $\operatorname{span}\left\{\left|c_{k}, c_{l}\right\rangle\right\}$ are considered, giving rise to 16 quadratic equations in the matrix elements of $A$. The solutions are (up to mode-permutations) all of the form $A=\mathbb{1}_{2} \oplus B$ with $B$ being a $2 \times 2$-matrix describing the interaction of the two logical- 1 modes while the others are just by-passed. Using the singular value decomposition, this matrix can be expressed as $B=V \operatorname{diag}\left(\sigma_{+}, \sigma_{-}\right) W$, where the unitaries $V$ and $W$ have immediate physical meaning: they describe beam splitters between the two modes. After rescaling the diagonal matrix by the largest singular value $\sigma_{+}$, an interpretation of $\operatorname{diag}\left(1, \sigma_{-} / \sigma_{+}\right)$can be given as well: One mode is left unaltered while the second one is damped by $\theta=\sigma_{-} / \sigma_{+}$. The success probability is then given by $p_{\mathrm{s}}=\sigma_{+}^{-4}$. It turns out [6] that for $\varphi \in[0, \pi]$ this optimal 


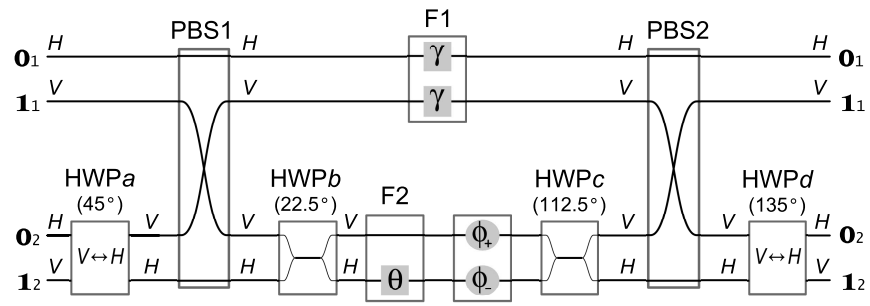

FIG. 1: Conceptual scheme of the gate. Vertically $(V)$ and horizontally $(H)$ polarized components of the same beam are drawn separately for clarity. In polarization beam splitters PBS1 and PBS2 the vertical components are reflected. Half-wave plates $\mathrm{HWP} b$ and $\mathrm{HWP} c$ act as "beam splitters" for $V$ and $H$ polarization modes. F1 and F2 are filters (attenuators), F1 acts on the both polarization modes, F2 on the $\mathrm{H}$ component only. Phase shifts $\phi_{+}$and $\phi_{-}$are introduced by proper path differences in the respective modes. HWP $a$ and HWP $d$ just swap vertical and horizontal polarizations. In the final setup they are omitted for simplicity and the second qubit is encoded inversely with respect to the first qubit.

success probability takes the form

$p_{\mathrm{s}}(\varphi)=\left(1+2\left|\sin \frac{\varphi}{2}\right|+2^{3 / 2} \sin \frac{\pi-\varphi}{4}\left|\sin \frac{\varphi}{2}\right|^{1 / 2}\right)^{-2}$

and $W=\left[\begin{array}{cc}-1 & -1 \\ 1 & -1\end{array}\right] / \sqrt{2}, V=W^{-1} \operatorname{diag}\left(\mathrm{e}^{\imath \phi_{+}}, \mathrm{e}^{\imath \phi_{-}}\right)$, where the phases in the lower Mach-Zehnder interferometer (between $\mathrm{HWP} b$ and $\mathrm{HWP} c$, see Fig.11) are defined by

$\phi_{ \pm}=\operatorname{arccot}\left[\cot \frac{\varphi+\pi}{4} \pm\left((2-2 \cos \varphi)^{1 / 4} \sin \frac{\varphi+\pi}{4}\right)^{-1}\right]$

Phase shifts are applied to each arm and one arm is damped by an attenuator (neutral-density filter) which is characterized by an amplitude transmissivity of

$$
\theta=\left(\frac{1+2 \sin \frac{\varphi}{2}-2(2-2 \cos \varphi)^{1 / 4} \cos \frac{\varphi+\pi}{4}}{1+2 \sin \frac{\varphi}{2}+2(2-2 \cos \varphi)^{1 / 4} \cos \frac{\varphi+\pi}{4}}\right)^{1 / 2}
$$

The remaining two attenuators in the upper beams are used to damp the amplitude of the by-passed modes to compensate for the overall losses in the lower beams. Their amplitude transmissivity reads $\gamma=p_{\mathrm{s}}^{1 / 4}$.

Details of the experiment: As the starting point of this experiment we generate a pair of photons in the process of type-I spontaneous parametric down-conversion. The laser beam of $250 \mathrm{~mW}$ of $\mathrm{cw}$ optical power emitted by Krypton-ion laser at $413 \mathrm{~nm}$ impinges on the $\mathrm{LiIO}_{3}$ crystal. Pairs of photons at $826 \mathrm{~nm}$ are collected using single mode fibres serving also as spatial filters. Subsequently, polarization controllers are employed to adjust the horizontal polarization of the photons.

The half-wave plates (HWP) and quarter-wave plates (QWP) in the input arms (see Fig. 2) are used to set the input states. Subsequently, the photons are superposed on the

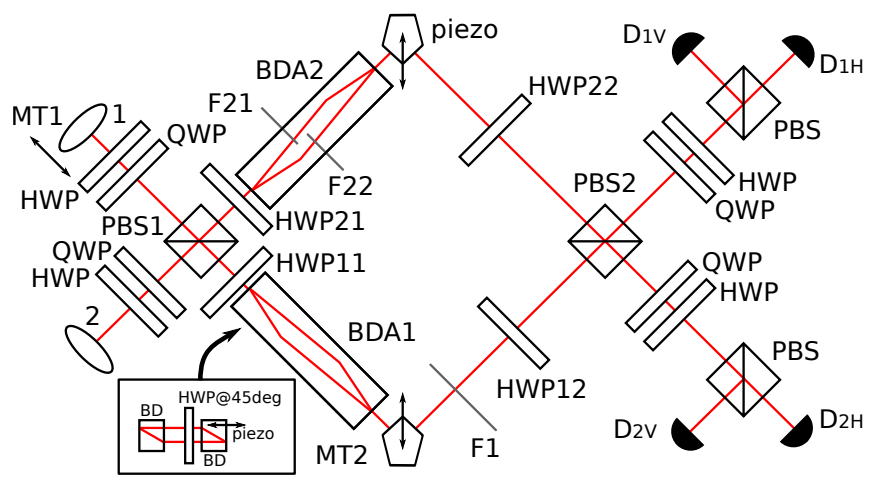

FIG. 2: Scheme of the actual experimental setup (see text for details).

first polarizing beam splitter PBS1 which transmits horizontal and reflects vertical polarization. Due to imperfections the transmissivity for horizontal polarization is only $95 \%$ (the remaining $5 \%$ are reflected). Polarization beam splitters also introduce parasitic phase shifts between vertical and horizontal polarization components. After leaving the PBS1 the photons in the upper arm are subjected to the action of half-wave plate HWP21. When set to $22.5 \mathrm{deg}$ it performs the transformation $|H\rangle \mapsto(|H\rangle+|V\rangle) / \sqrt{2},|V\rangle \mapsto(|H\rangle-|V\rangle) / \sqrt{2}$, where $|H\rangle$ and $|V\rangle$ denote horizontal and vertical polarization states, respectively. The lower arm is also equipped with a half wave-plate (HWP11) but it is set to zero (its presence just guarantees the same optical paths, dispersion effects, etc. in the both arms). Behind the wave plates there are the beamdivider assemblies BDA1 and BDA2. They consist of two beam dividers (BD) splitting and subsequently rejoining horizontal and vertical polarizations. BDA2 is equipped with gradient neutral-density filters F21 and F22 (see Fig. 2). This way one can perform arbitrary polarization sensitive losses. BDA1 is used just to equilibrate the beam position and the optical length of the both arms. After leaving the beam-divider assemblies the photons propagate through half-wave plates HWP12 and HWP22. HWP22 is set to 22.5 deg reversing thus the transformation imposed by HWP21. HWP12 is set to $45 \mathrm{deg}$ to compensate for the polarization flip between the $H$ and $V$ polarizations performed by BDA1. The lower arm is equipped with a gradient neutral density filter F1 to apply polarization independent losses. The gate operation itself is completed by overlapping the photons on the second polarizing beam splitter PBS2. To be able to perform complete state and thereby process tomography we employ polarization analysis in the both output arms. The analysis consists of QWPs and HWPs followed by polarizing beam splitters, cut-off filters and single mode fibres leading to single photon detectors.

Gate operation: The setup is then adjusted to perform the gate operation. First we set filters F21 and F1 to introduce the required losses. After that the wave plates HWP21 and HWP22 are set to $22.5 \mathrm{deg}$. The phase in the beam divider assembly BDA2 is set to maximize the visibility of the interferometer formed by PBS1 and PBS2. The precise tuning of 


\begin{tabular}{cccccccc}
\hline \hline$\varphi$ & $F_{\chi}$ & $F_{\mathrm{av}}$ & $F_{\min }$ & $\mathcal{P}_{\mathrm{av}}$ & $\mathcal{P}_{\min }$ & $p_{\mathrm{s}, \mathrm{obs}}$ & $p_{\mathrm{s}, \mathrm{th}}$ \\
\hline 0 & 0.94 & 0.96 & 0.84 & 0.96 & 0.87 & $0.87 \pm 0.08$ & 1.00 \\
$0.05 \pi$ & 0.95 & 0.96 & 0.91 & 0.96 & 0.87 & $0.37 \pm 0.05$ & 0.36 \\
$0.125 \pi$ & 0.91 & 0.90 & 0.77 & 0.95 & 0.87 & $0.19 \pm 0.03$ & 0.21 \\
$0.25 \pi$ & 0.84 & 0.88 & 0.73 & 0.90 & 0.67 & $0.11 \pm 0.02$ & 0.13 \\
$0.5 \pi$ & 0.86 & 0.89 & 0.81 & 0.90 & 0.76 & $0.09 \pm 0.01$ & 0.09 \\
$0.75 \pi$ & 0.84 & 0.87 & 0.63 & 0.90 & 0.71 & $0.08 \pm 0.01$ & 0.09 \\
$\pi$ & 0.84 & 0.86 & 0.71 & 0.92 & 0.83 & $0.12 \pm 0.01$ & 0.11 \\
\hline \hline
\end{tabular}

TABLE I: Process fidelities $\left(F_{\chi}\right)$, average $\left(F_{\mathrm{av}}\right)$ and minimal $\left(F_{\min }\right)$ output-state fidelities, average $\left(\mathcal{P}_{\text {av }}\right)$ and minimal $\left(\mathcal{P}_{\text {min }}\right)$ outputstate purities and actually observed ( $\left.p_{\mathrm{s}, \mathrm{obs}}\right)$ and theoretically predicted $\left(p_{\mathrm{s}, \mathrm{th}}\right)$ success probabilities for different phases $(\varphi)$.
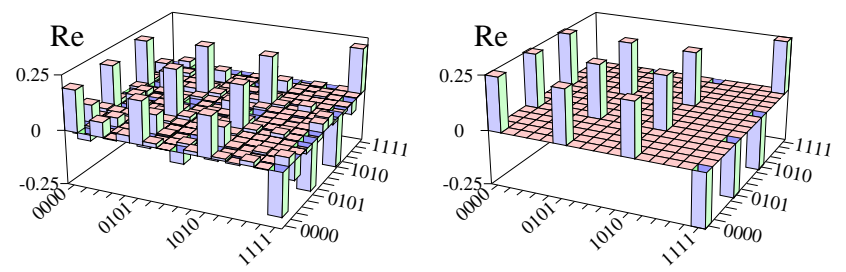

FIG. 3: Matrix representation of the CP map characterizing the operation of the controlled-phase gate with $\varphi=\pi$. The left panel shows the real part of the reconstructed process matrix, the right one displays the real part of the ideal theoretical $\mathrm{CP}$ map. Imaginary parts are negligible (zero in ideal case). The process fidelity $F_{\chi}=84 \%$.

the gate is then performed by switching between the inputs $\left|H_{1}, R_{2}\right\rangle$ and $\left|V_{1}, R_{2}\right\rangle$, where indices 1 and 2 denote the input modes and $R$ stands for the right circular polarization. Using the circular detection basis in the second output arm we can observe the phase applied by the gate when the polarization of the first input photon flips from $|H\rangle$ to $|V\rangle$. In this configuration we also tune the phase shift inside the beam divider assembly BDA2 and the phase shift between the two arms of the Mach-Zehnder interferometer formed by PBS1 and PBS2.

Results: Gradually we have adjusted the gate to apply 7 phases in the range between 0 and $\pi$. Each time we have performed complete process tomography and estimated the process matrix using the maximum likelihood method. Fidelities of the process lie in the range from $84 \%$ to $95 \%$ (see Tab. (I). Figs. 3 and 4 show examples of experimentally obtained process matrices and their theoretical counterparts for $\varphi=\pi$ and $\pi / 2$.

For each selected phase we simultaneously measured twophoton coincidence counts between detectors $\mathrm{D}_{1 \mathrm{H}} \& \mathrm{D}_{2 \mathrm{H}}$, $\mathrm{D}_{1 \mathrm{~V}} \& \mathrm{D}_{2 \mathrm{~V}}, \mathrm{D}_{1 \mathrm{H}} \& \mathrm{D}_{2 \mathrm{~V}}$, and $\mathrm{D}_{1 \mathrm{~V}} \& \mathrm{D}_{2 \mathrm{H}}$, each for $3 \times 3 \mathrm{com}-$ binations of polarization measurement bases in the output arms. This amounts to measuring projections onto horizontal/vertical, diagonal/anti-diagonal and right/left circular polarizations. The diagonal and anti-diagonal linear polarization states are defined as $|D\rangle=(|H\rangle+|V\rangle) / \sqrt{2}$ and $|A\rangle=(|H\rangle-$ $|V\rangle) / \sqrt{2}$ and the right- and left-handed circular polarization
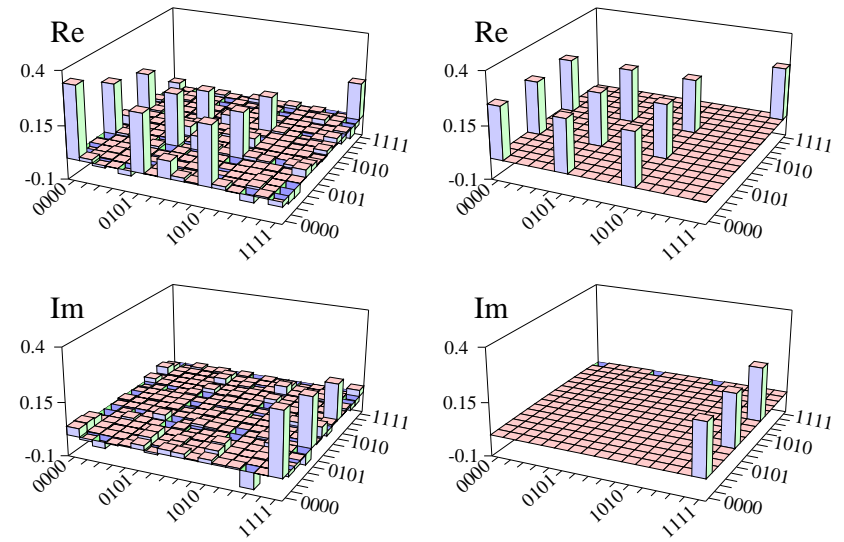

FIG. 4: Choi matrices for the gate with $\varphi=\pi / 2$. The left top panel shows the real part of the reconstructed process matrix while the left bottom one displays its imaginary part. The process fidelity $F_{\chi}=86 \%$. The two right panels show the ideal matrix.

states read $|R\rangle=(|H\rangle+i|V\rangle) / \sqrt{2},|L\rangle=(|H\rangle-i|V\rangle) / \sqrt{2}$. The unequal detector efficiencies were compensated by proper re-scaling of the measured coincidence counts [10]. Each measurement was done for 36 different input product states. Namely, for $6 \times 6$ combinations of polarization state vectors $|H\rangle,|V\rangle,|D\rangle,|A\rangle,|R\rangle$, and $|L\rangle$ of each input photon. This complex measurement provided us with tomographically complete data enabling us to fully characterize the implemented operation by quantum process tomography [11, 12] as well as to reconstruct density matrices of output states for each used input state.

Active stabilization: Each setting of an input and output polarization basis was preceded by an active stabilization. For the purpose of the stabilization the fixed input state and output detection basis were always used. In this setting the visibility in the interferometer formed by PBS1 and PBS2 was measured. If this visibility was lower than a selected threshold then the positions of MT1 (interferometer lengths) and MT2 (dip position) were optimized and the phase drift was compensated. Finally the required polarizations were set and data were accumulated within $5 \mathrm{~s}$.

Process tomography: Any quantum operation can be fully described by a completely positive map and-according to the Jamiolkowski-Choi isomorphism-represented by a positivesemidefinite operator $\chi$ on the tensor product of input and output Hilbert spaces [13]. In our case $\chi$ is a $16 \times 16$ square matrix. From the measured data we can reconstruct $\chi$ for any setting of $\varphi$ using maximum likelihood estimation [12, 14]. To quantify the quality of the operation we calculate the process fidelity, if $\chi_{\mathrm{id}}$ is a one-dimensional projector its common definition is $F_{\chi}=\operatorname{Tr}\left[\chi \chi_{\mathrm{id}}\right] /\left(\operatorname{Tr}[\chi] \operatorname{Tr}\left[\chi_{\mathrm{id}}\right]\right)$. Here $\chi_{\mathrm{id}}$ represents the ideal transformation corresponding to the controlled-phase gate. Specifically,

$$
\chi_{\mathrm{id}}=\sum_{i, j, k, l=V, H}|i, j\rangle\langle k, l|\otimes U| i, j\rangle\langle k, l| U^{\dagger},
$$




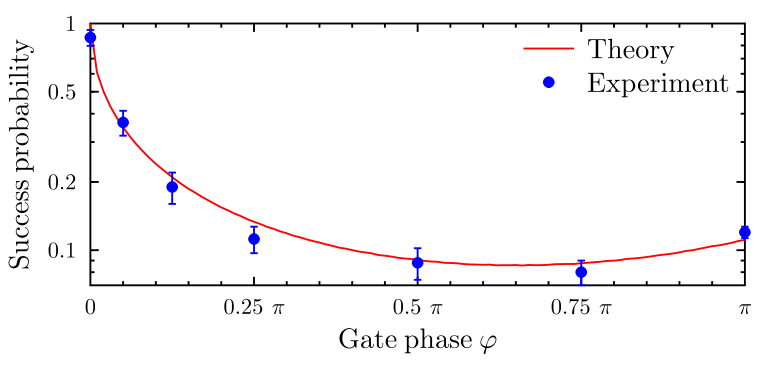

FIG. 5: Success probability of the gate.

where $U$ stands for the unitary operator on two qubits defined by Eq. (1). We have also reconstructed the density matrices of output two-photon states corresponding to all product inputs $|j, k\rangle, j, k \in\{H, V, D, A, R, L\}$. This was done for all values of $\varphi$. An important parameter characterizing the gate performance is the fidelity of output states $\rho_{\text {out }}$ defined as $F=\left\langle\psi_{\text {out }}\left|\rho_{\text {out }}\right| \psi_{\text {out }}\right\rangle$, where $\left|\psi_{\text {out }}\right\rangle=U\left|\psi_{\text {in }}\right\rangle$ and $\left|\psi_{\text {in }}\right\rangle$ is the input state vector. Table \contains the average and minimal values of state fidelities for different phases. Fidelities $F_{\text {av }}$ are averaged over all output states corresponding to our 36 input states; $F_{\min }$ denote minimal values. Another important characteristics is the purity of the output state $\rho_{\text {out }}$, defined as $\mathcal{P}=\operatorname{Tr}\left[\rho_{\text {out }}^{2}\right]$. If the input state is pure the output state is expected to be pure as well. The average and minimal purities of output states are also given in Table [

Trade off in success probabilities: The most important result of this paper-aside from the technological implications - is the experimental verification of the trade-off between the phase shift applied by the gate and the corresponding success probability of the gate. We have estimated the success probability for each value of the selected phase shifts. It was calculated as a ratio of the number of successful gate operations per time interval and the number of reference counts during the same interval (measured with no filters and with the wave plates set to 0 ). We have determined the success probability for the all selected input states. These probabilities were averaged and the standard deviations were calculated. Notice that the calibration measurements collect coincidence counts behind the setup (using the same detectors as in the subsequent measurements), thus all the "technological" losses in the setup and low detector efficiencies are included in the calibration. Therefore the estimated success probabilities are not burdened by these "technological" losses. They can be compared with the theoretical predictions in Tab. I and in Fig. 5. One can see a very good agreement with the theoretical prediction.

Conclusions: We have built the first implementation of the tunable linear-optical controlled phase gate which is optimal for any value of the phase shift. Changing the parameters of the setup the gate can apply any phase shift from the interval $[0, \pi]$ on the controlled qubit. We have thoroughly tested the performance of the gate using full quantum process tomography. Obtained process fidelities range from $84 \%$ to $95 \%$. We have also experimentally verified that all our controlled phase gates are optimal in the sense that they operate at the maximum possible success probabilities that are achievable by linear-optical setups. The experimental verification of this trade-off between the phase shift applied by the gate and the corresponding success probability of the gate is the most notable result of our work. It demonstrates the contra-intuitive fact that the optimal success probability is not monotonous with the phase shift increasing from 0 to $\pi$. It is the hope that the flexible tool established here proves useful in devising further optical linear optical circuits for optical quantum information processing and that ideas developed in this work find their way to realization in fully integrated optical architectures.

Acknowledgments: This work was supported by the Czech Ministry of Education (1M06002, MSM6198959213), the Czech Science Foundation (202/09/0747), the EU (QESSENCE, MINOS, COMPAS), the EURYI and the Palacky University (PrF-2010-009 and PrF-2010-020).

[1] E. Knill et al., Nature 409, 46 (2001).

[2] W. J. Munro et al., J. Opt. B 7, S135 (2005); J. L. O’Brien, Science 318, 1567 (2007); I. A. Walmsley, Science 319, 1211 (2008); M. Aspelmeyer and J. Eisert, Nature 455, 180 (2008); A. Politi et al., Science 320, 646 (2008); B. J. Smith et al., arXiv:0905.2933.

[3] M. A. Nielsen and I. L. Chuang, Quantum computation and quantum information (Cambridge University Press, 2000).

[4] H.F. Hofmann and S. Takeuchi, Phys. Rev. A 66, 024308 (2002); N. K. Langford et al., Phys. Rev. Lett. 95, 210504 (2005); N. Kiesel et al., ibid. 95, 210505 (2005).

[5] B. P. Lanyon et al., Nat. Phys. 5, 134 (2009).

[6] K. Kieling, J. O’Brien, and J. Eisert, New J. Phys. 12, 013003 (2010).

[7] W. Wieczorek et al., Phys. Rev. Lett. 101, 010503 (2008).

[8] S. Scheel, arXiv:quant-ph/0406127

[9] K. Kieling, PhD thesis, Imperial College London (2008).

[10] J. Soubusta et al., Phys. Rev. A 76, 042318 (2007).

[11] J. F. Poyatos et al., Phys. Rev. Lett. 78, 390 (1997); I. L. Chuang and M. A. Nielsen, J. Mod. Opt. 44, 2455 (1997); J. Fiurášek and Z. Hradil, Phys. Rev. A 63, 020101 (2001); M. F. Sacchi, ibid. 63, 054104 (2001).

[12] M. Ježek et al., Phys. Rev. A 68, 012305 (2003).

[13] A. Jamiołkowski, Rep. Math. Phys. 3, 275 (1972); M.-D. Choi, Lin. Alg. Appl. 10, 285 (1975).

[14] M. G. A. Paris and J. Řeháček (Eds.), Quantum State Estimation, Lect. Notes Phys. 649, (Springer, Berlin, Heildeberg, 2004). 